\title{
Field evaluation of plant extracts for the control of diamond moth (Plutella xylostella Linnaeus) infesting Cabbage (Brassica oleracea Linn)
}

\author{
Adeola Foluke Odewole*, Timothy Abiodun Adebayo \\ Department of Crop and Environmental Protection, Ladoke Akintola University of Technology, \\ P.M.B. 4000, Ogbomoso 210001, Nigeria \\ `E-mail address: ogunkeyede@yahoo.com , aodewole@lautech.edu.ng
}

\begin{abstract}
Cabbage (Brassica oleracea Linn.) is one of the important vegetables grown worldwide. Insect pests have been a major constraint to its production with some reported to have developed resistance to commonly available synthetic insecticides. In order to find alternative control for these pests, field experiments were conducted in the early seasons of 2011 and 2012, to evaluate the efficacy of three plant extracts, Tephrosia vogelli Hooks, Zingiber officinales Rosh and Lantana camara Spanish flag, applied at 5, 10 and $25 \% \mathrm{w} / \mathrm{v}$ concentrations Delthamethrin (a synthethic insecticide) and an unsprayed plot were included as positive and negative controls respectively. Plots were arranged in a Randomized Complete Block Design (RCBD), with three replications. Mean Data over the two planting seasons were subjected to the analysis of variance and significant means were compared using the least significant difference (L.S.D) at $\mathrm{P}<0.05$. Z. officinales extract effectively control $P$. xylostella larvae at all concentrations with $25 \% \mathrm{w} / \mathrm{v}$ gave the best yield of $0.50 \mathrm{~kg} / \mathrm{ha}$ while $T$. vogelii and $L$. camara extracts were as effective as Deltamethrin at different concentrations and hours on diamondback-moth ( $P$. xylostella) adult The present findings indicated that effective botanicals against Diamondback moth adult and larvae of cabbage can be formulated from extract of $T$. vogelii, $L$. camara and $Z$. officinales at $25 \%$ concentration respectively.
\end{abstract}

Keywords: Cabbage; Deltamethrin; Plutella xylostella; Z. officinales; L. camara; T. vogelii

\section{INTRODUCTION}

Cabbage is a popular cultivar of the species Brassica oleracea Linn. of the family Brassicaceae. It is one of the most important leafy vegetables worldwide and is a crop grown by smallholder farmers in Africa (Talaker, 2000). It has medicinal properties and it is an excellent source of vitamin $\mathrm{C}$, minerals, dietary fibers, folates and especially phytochemicals (Jagerstad et al., 2004: Kyung Young Yoon et al., 2006: Rodriguez et al. 2006). Cabbage also reduces risk of heart disease and stroke, alleviate rheumatisms and skin problems. Fresh cabbage juice has been shown to promote rapid healing of peptic ulcer (USDA 2009).

Despite the long time of Cabbage production in Africa, it still faces several constraints including insect pests. These insect pests includes diamondback moth (Plutela xylostella), Cabbage looper (Tricoplusia ni), Cabbage root maggot (Delia radicum), Cabbage webworm 
(Crocidolomia Pavonana), Common cutworm (Spodoptera litura), Cabbage Borer (Hellula undalis), Cabbage aphids (Brevicoryne brassicae), imported cabbage worm (Pieris rapae), cutworm (Agrotis sp) and Cabbage flea beetle (Phyllotreta cruciderae) (Talekar, 1992). These pests can cause serious damage to young transplants and old plants. Damage to the head or wrapper leaves often reduces marketability, hence, there is the need to control insect pests of cabbage.

Management of the pests is heavily reliant on the use of chemical insecticides especially in the developing countries of the tropics (Tabashmik et al.,1987; Dandang et al, 2003a). Synthetic insecticides have been reported to have some side effects such as mammalian toxicity, environmental pollution, insect resistance and resurgence high cost and unavailability at critical periods (Duke, 1990). Arising from these highlighted problems, there is the need to search for alternatives for synthetic insecticides. Such agents should be pestspecific, non-toxic to humans and other biota, biodegradable, less prone to pest resistance and resurgence, relatively less expensive and with relative ease of application (Devlin and Zettel, 1999; Adebayo, 2003).

The basis for this research is an attempt to solve some of the problems facing farmers, like problem of low productivity due to the insect pests attack. Protecting consumers from eating pesticide-contaminated vegetables and exposure of farmers to the toxic synthetic chemicals. Therefore, the objective of this research is to determine the effect of Tephrosia vogelli Hooks, Zingiber officinales Rosh and Lantana camara L. extracts on two developmental stages of $P$. xylostella infesting cabbage insect pest diversity and population, head damage and yield of cabbage.

\section{MATERIALS AND METHODS}

\section{1. Experimental site}

Field study was conducted at Ladoke Akintola University of Technology (LAUTECH) Teaching and Research Farm, Ogbomoso, Nigeria. Ogbomoso is located on longitude $4^{\circ} 30^{\prime}$ and latitude $10^{\circ} 5^{\prime} \mathrm{N}$. The climate of the region could be described as hot humid tropical falls in Southern Guinea Savanna of Nigeria with a mean temperature of $27^{\circ} \mathrm{C}$ and annual rainfall of $1400 \mathrm{~mm}$, marked with dry and wet seasons and characterized by a bi-modal rainfall pattern with peaks in July and September.

\section{2. Experimental materials}

The seed of cabbage (var. Copanheng) was obtained from the Seed Project Company Limited Kano, Nigeria. while ginger rhizome was purchased from Sabo Market Ogbomoso Nigeria. Matured leaves of T. vogelii, L. camara were collected from the premises of the Department of Crop and Environmental Protection, LAUTECH, Ogbomoso, Nigeria. Deltamethrin was purchased from Farmers Chemical Agro allied Shop Ogbomoso.

\section{3. Preparation of plant extract}

Fresh leaves of L. camara and T. vogelii; and rhizome of Z. officinales were harvested at full maturity. The extracts of the three plants species were prepared by weighing $1000 \mathrm{~g}$ each of botanical separately and crushed with a mortar and pestle. The crushed plant parts were put in separate plastic buckets containing two liters of water. These were allowed to 
settle for 24 hours and the aqueous suspension was filtered through muslin cloth. The filtrate was stored in plastic containers as stock solution for bioassay and kept in the refrigerator until use.

\section{4. Agronomic practices}

The Experimental site was manually cleared and divided into - plots. Each plot was divided into beds having $2 \mathrm{~m} \times 3 \mathrm{~m}$ dimension with $1 \mathrm{~m}$ gap between adjacent plots. A gap of $2 \mathrm{~m}$ was used to separate different blocks. A total of thirty three (33) plots were made to accommodate the treatments. Each plot had 5 rows with 6 plants per row spaced out at $0.6 \mathrm{~m}$ x $0.5 \mathrm{~m}$. which amounted to 30 plants per plot and 50,000 plants per hectare. The plants were Four plant stands from the middle rows were tagged for data collection.

Cabbage seeds were planted in early season of 2011 and 2012 on 12 May 2011 and 23 May 2012 respectively at the rate of three to four seeds per stand. Thinning was done two weeks after planting (WAP) to achieve one plant per stand. Weeding was done as at when due.

From the stock solutions, 50, 100 and $250 \mathrm{ml}$ each of the solution was diluted with 1000 $\mathrm{ml}$ of water to have 5, 10 and $25 \%$ concentration. The unsprayed plots and synthetic insecticides (Deltamethrin used at the rate of 0.8 litres /hectare) were included for comparison. The treatments were applied by spraying the plants very early in the morning on weekly basis. This was done according to the method of Owolade et al. (2004) and Oparake (2005) in order to reduce or eliminate pesticide drift. Foliar application was done using hand sprayer which commenced 4 WAP and was repeated at weekly intervals which were made weekly over a 4 - week period given a total of four applications.

\section{5. Data collection and Data Analysis}

Data were collected on insect pest population, level of damaged caused by the insect pest, vegetative, head damage and yield parameters. Data collected over the two years were pooled and subjected to analysis of variance (ANOVA) using Randomized Complete Block Design (RCBD) according to by Gomez and Gomez (1987). Significant means were compared using least significant difference (LSD) at $5 \%$ probability level.

\section{RESULTS}

Z. officinales extract was significantly better at $(p=0.05)$ in the control of P. xylostella larvae with mean number of $0.71 \pm 0.00$ compared with Deltamethrin $(1.71 \pm 0.34)$. The best control against $P$. xylostella larvae was observed at $25 \%$ concentrations w/v. Application of $T$. vogelii and $L$. camara extracts were as effective as Deltamethrin at different concentrations on diamondback-moth ( $P$. xylostella) adult. The mean number of $P$. xylostella adult on $T$. vogelli and $L$. camara were $(0.91 \pm 0.20,0.76 \pm 0.05,0.71 \pm 0.000 .91 \pm 0.20,0.76 \pm 0.05,0.76$ \pm 0.05 and $0.85 \pm 0.14$ at 5.10 and $25 \%$ concentrations respectively. The efficacy increased with repeated spraying across the treatments. With respect to hours after spraying as hours increases, rate of insect pest decreases. Plant height increased with increase in concentrations, with $Z$. officinales having the highest mean value $(22.71 \pm 1.74 \mathrm{~cm})$ at $25 \% \mathrm{w} / \mathrm{v}$ concentrations followed by $T$. vogelii $(21.44 \pm 0.34 \mathrm{~cm})$ at the same concentration. However, as the concentrations of plant extracts increased, the rate of leaf damage decreased with the exception of the plot treated with Z. officinales, L. camara - treated plot at $25 \%(\mathrm{w} / \mathrm{v})$ 
concentrations had the least mean value $(1.25 \pm 0.14)$ of head damage and $Z$. officinales at $25 \%$ concentrations gave the best yield.

Table 1. Effect of botanicals on Diamondback moth (Plutella xylostella) population at $1^{\text {st }}$ spraying.

\begin{tabular}{|c|c|c|c|c|c|c|c|}
\hline \multirow[t]{2}{*}{ TREATMENTS } & \multicolumn{7}{|l|}{ CONCENTRATIONS } \\
\hline & $(\%)$ & 崖 & 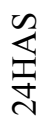 & 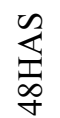 & 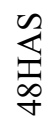 & $\underset{⿱ \mathbb{N}}{\stackrel{\mathbb{N}}{\mathbb{N}}}$ & $\underset{\mathbb{N}}{\stackrel{\mathbb{S}}{\mathbb{N}}}$ \\
\hline & & $\underset{\stackrel{m}{0}}{\stackrel{B}{B}}$ & 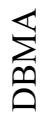 & $\sum_{\stackrel{D}{0}}^{+}$ & 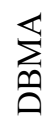 & $\sum_{\overrightarrow{0}}^{\vec{D}}$ & 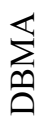 \\
\hline Control & unsprayed & $\begin{array}{l}0 \\
0 \\
0 \\
+1 \\
\infty \\
0\end{array}$ & $\begin{array}{l}n \\
0 \\
0 \\
+ \\
0 \\
0 \\
0\end{array}$ & $\begin{array}{l}\cong \\
\Xi \\
+ \\
\infty \\
\infty \\
0 \\
0\end{array}$ & $\begin{array}{l}8 \\
8 \\
0 \\
\text { H } \\
0 \\
0\end{array}$ & $\begin{array}{l}8 \\
\dot{0} \\
\ddot{H} \\
\stackrel{0}{0}\end{array}$ & $\begin{array}{l}\stackrel{8}{0} \\
\stackrel{0}{+} \\
\stackrel{1}{*} \\
0\end{array}$ \\
\hline Z. officinales & 5 & $\begin{array}{l}0 \\
0 \\
0 \\
+ \\
\infty \\
0\end{array}$ & 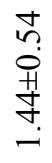 & $\begin{array}{l}0 \\
0 \\
0 \\
+1 \\
0 \\
0\end{array}$ & $\begin{array}{l}\infty \\
\text { ?. } \\
\stackrel{+}{+} \\
\stackrel{+}{+} \\
\end{array}$ & 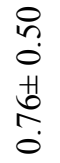 & 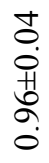 \\
\hline L. camara & 5 & 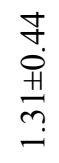 & $\begin{array}{l} \pm \\
\dot{0} \\
+ \\
\infty \\
\infty \\
0\end{array}$ & 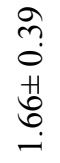 & $\begin{array}{l}\text { ㄱ. } \\
\dot{8} \\
\stackrel{+}{0} \\
\stackrel{\Omega}{0}\end{array}$ & $\begin{array}{l}8 \\
0 \\
\dot{0} \\
\stackrel{0}{0} \\
\vdots \\
0\end{array}$ & 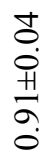 \\
\hline T. vogelii & 5 & $\begin{array}{l}0 \\
0 \\
0 \\
+1 \\
0 \\
0 \\
0\end{array}$ & 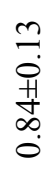 & $\begin{array}{l}8 \\
0 \\
0 \\
\text { ㅍ } \\
\text { ‥ }\end{array}$ & $\begin{array}{l} \pm \\
\pm \\
0 \\
\text { H } \\
\infty \\
0 \\
0\end{array}$ & $\begin{array}{l}8 \\
0 \\
+ \\
+1 \\
\vdots \\
0\end{array}$ & 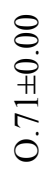 \\
\hline Z. officinales & 10 & $\begin{array}{l}8 \\
\dot{0} \\
\stackrel{+}{\#} \\
0 \\
0\end{array}$ & $\begin{array}{l}0 \\
\vdots \\
0 \\
+1 \\
\infty \\
0\end{array}$ & $\begin{array}{l}0 \\
0 \\
0 \\
+1 \\
0 \\
0\end{array}$ & $\begin{array}{l}\text { o. } \\
\dot{0} \\
+1 \\
0 \\
0\end{array}$ & 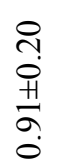 & $\begin{array}{l}0 \\
\stackrel{0}{0} \\
\stackrel{1}{1} \\
\infty \\
0 \\
0\end{array}$ \\
\hline L. camara & 10 & $\begin{array}{l}\vec{b} \\
0 \\
+ \\
\sim \\
\tilde{O}\end{array}$ & $\begin{array}{l} \pm \\
\\
+ \\
\infty \\
\infty \\
\infty \\
0\end{array}$ & $\begin{array}{l}\text { f. } \\
\text { o } \\
\text { 1 } \\
\infty \\
ت\end{array}$ & $\begin{array}{l}\tilde{N} \\
0 \\
+ \\
+ \\
\vdots \\
0\end{array}$ & $\begin{array}{l}\stackrel{0}{1} \\
0 \\
+ \\
\vdots \\
\vdots\end{array}$ & $\begin{array}{l}\infty \\
0 \\
0 \\
0 \\
+ \\
0 \\
0 \\
0\end{array}$ \\
\hline T. vogelii & 10 & 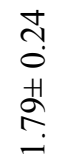 & 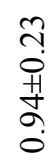 & $\begin{array}{l}0 \\
\\
0 \\
+1 \\
0 \\
0 \\
0 \\
-\end{array}$ & $\begin{array}{l}\text { I. } \\
\dot{0} \\
+ \\
\infty \\
\infty \\
0\end{array}$ & $\begin{array}{l}\infty \\
n \\
0 \\
+ \\
\text { †े } \\
\text { In }\end{array}$ & 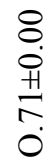 \\
\hline Z. officinales & 25 & 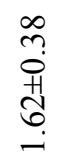 & $\begin{array}{l}\text { oे } \\
\text { ○ } \\
+1 \\
+ \\
\infty\end{array}$ & 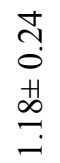 & 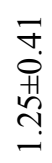 & $\begin{array}{l}\vec{ก} \\
0 \\
+ \\
\text { + } \\
\stackrel{0}{0}\end{array}$ & $\begin{array}{l}n \\
0 \\
0 \\
+1 \\
0 \\
0\end{array}$ \\
\hline
\end{tabular}




\begin{tabular}{|c|c|c|c|c|c|c|c|}
\hline L. camara & 25 & $\begin{array}{l}\vec{m} \\
0 \\
+ \\
m \\
m\end{array}$ & 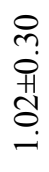 & $\begin{array}{l}\infty \\
\stackrel{+}{0} \\
+ \\
+ \\
\infty \\
\infty \\
-\end{array}$ & 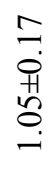 & $\begin{array}{l}\vec{N} \\
0 \\
+1 \\
8 \\
8\end{array}$ & $\begin{array}{l}\infty \\
0 \\
0 \\
0 \\
0 \\
\infty \\
0 \\
0\end{array}$ \\
\hline T. vogelii & 25 & 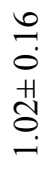 & $\begin{array}{l}0 \\
0 \\
0 \\
+1 \\
\infty \\
0 \\
0\end{array}$ & $\begin{array}{l} \pm \\
\dot{0} \\
H \\
\tilde{D} \\
0 \\
0\end{array}$ & $\begin{array}{l}8 \\
\stackrel{0}{0} \\
\dot{H} \\
\stackrel{1}{0} \\
\dot{0}\end{array}$ & $\begin{array}{l} \pm \\
\text { 芫 } \\
n \\
0\end{array}$ & 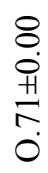 \\
\hline Deltamethrin & 0.04 & 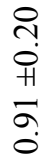 & $\begin{array}{l}n \\
0 \\
0 \\
+1 \\
\infty \\
0 \\
0\end{array}$ & $\begin{array}{l}0 \\
0 \\
0 \\
+1 \\
0 \\
0\end{array}$ & $\begin{array}{l}8 \\
0 \\
\dot{0} \\
+1 \\
\ddot{0} \\
0\end{array}$ & 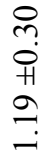 & $\begin{array}{l}n \\
0 \\
0 \\
\text { Hु } \\
0 \\
0\end{array}$ \\
\hline $\operatorname{LSD}(5 \%)$ & & $\begin{array}{l}\bar{b} \\
0\end{array}$ & $\stackrel{+}{+}$ & $\begin{array}{l}\widetilde{\sigma} \\
0\end{array}$ & $\begin{array}{l}\text { ণे } \\
\text { ָे }\end{array}$ & ñ & $\stackrel{0}{\stackrel{0}{0}}$ \\
\hline
\end{tabular}

Values are mean $( \pm$ SEM $)(n=3)$. LSD values are applicable along the column. Delthamethrin $(0.81 /$ ha $)$, $\mathrm{DBML}=$ Diamondback moth larva, DMBA $=$ Diamondback-moth Adult, HAS $=$ Hours after spraying.

Table 2. Effect of botanicals on Diamondback moth (Plutella xylostella) population at $2^{\text {nd }}$ spraying.

\begin{tabular}{|c|c|c|c|c|c|c|c|}
\hline TREATMENTS & CONCENTRATIONS & & & & & & \\
\hline & $(\%)$ & 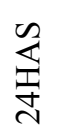 & $\begin{array}{l}\underset{J}{J} \\
\underset{\sim}{ \pm}\end{array}$ & 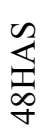 & $\begin{array}{l}\underset{1}{4} \\
\underset{+}{\infty}\end{array}$ & $\underset{\mathbb{N}}{\stackrel{\mathbb{N}}{\mathbb{N}}}$ & $\begin{array}{l}\mathbb{Z} \\
\mathbb{N} \\
\mathbb{N}\end{array}$ \\
\hline & & $\underset{⿱ 乛}{\stackrel{B}{\prime}}$ & 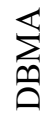 & 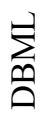 & 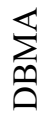 & $\sum_{\overrightarrow{0}}$ & 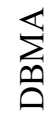 \\
\hline Control & Unsprayed & 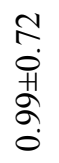 & $\begin{array}{l}8 \\
\circ \\
+1 \\
\text { ㅇ. } \\
0\end{array}$ & $\begin{array}{l} \pm \\
\stackrel{0}{0} \\
+1 \\
\text { मे } \\
\infty \\
0\end{array}$ & 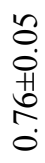 & 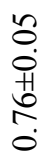 & $\begin{array}{l}8 \\
\dot{0} \\
\dot{+} \\
\frac{1}{1} \\
\dot{0}\end{array}$ \\
\hline Z. officinales & 5 & $\begin{array}{l}\hat{\sigma} \\
0 \\
+ \\
\infty \\
\vec{i} \\
\text { i }\end{array}$ & $\begin{array}{l}\underset{+}{0} \\
+ \\
\hat{+} \\
\end{array}$ & $\begin{array}{l}\stackrel{8}{0} \\
\dot{0} \\
\dot{H} \\
\infty \\
0\end{array}$ & $\begin{array}{l}2 \\
0 \\
0 \\
\text { H } \\
\infty \\
0\end{array}$ & 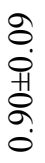 & $\begin{array}{l}\text { o. } \\
\dot{0} \\
+ \\
\infty \\
0 \\
0\end{array}$ \\
\hline L.camara & 5 & 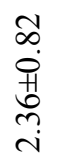 & 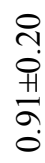 & 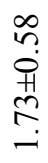 & $\begin{array}{l}\stackrel{8}{0} \\
0 \\
+ \\
\infty \\
0\end{array}$ & 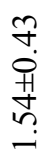 & $\begin{array}{l}m \\
\ddot{0} \\
\dot{+} \\
\dot{\sigma} \\
0\end{array}$ \\
\hline
\end{tabular}




\begin{tabular}{|c|c|c|c|c|c|c|c|}
\hline T. vogelii & 5 & $\begin{array}{l}\frac{m}{0} \\
\frac{\dot{H}}{0} \\
0\end{array}$ & $\begin{array}{l}\stackrel{0}{1} \\
\vdots \\
+1 \\
\vdots \\
0\end{array}$ & 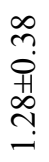 & $\begin{array}{l}\infty \\
0 \\
0 \\
0 \\
0 \\
0 \\
0 \\
0\end{array}$ & $\begin{array}{l}\infty \\
- \\
0 \\
0 \\
0 \\
0\end{array}$ & $\begin{array}{l}8 \\
0 \\
0 \\
+1 \\
\vdots \\
0\end{array}$ \\
\hline Z. officinales & 10 & 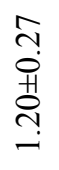 & 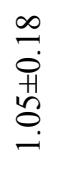 & $\begin{array}{l}\infty \\
0 \\
0 \\
\dot{0} \\
0 \\
\infty \\
0 \\
0\end{array}$ & $\begin{array}{l}8 \\
\stackrel{0}{0} \\
\stackrel{+}{*} \\
\dot{0}\end{array}$ & $\begin{array}{l}2 \\
0 \\
0 \\
+1 \\
\stackrel{0}{0} \\
0\end{array}$ & $\begin{array}{l}n \\
0 \\
0 \\
+1 \\
\stackrel{0}{0} \\
\dot{0}\end{array}$ \\
\hline L.camara & 10 & $\begin{array}{l}\mathfrak{n} \\
0 \\
+ \\
n \\
n\end{array}$ & 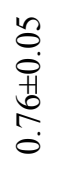 & 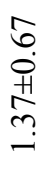 & $\begin{array}{l}\infty \\
\stackrel{0}{0} \\
\dot{+} \\
0\end{array}$ & $\begin{array}{l}\vec{N} \\
\text { on } \\
\text { ò } \\
0\end{array}$ & $\begin{array}{l}8 \\
\dot{0} \\
\dot{+} \\
\frac{1}{0} \\
\dot{0}\end{array}$ \\
\hline T. vogelii & 10 & 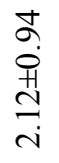 & 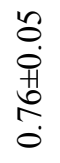 & 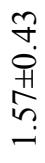 & 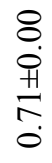 & $\begin{array}{l}n \\
n \\
0 \\
\text { 1n } \\
\text { ô }\end{array}$ & 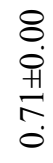 \\
\hline Z. officinales & 25 & $\begin{array}{l}8 \\
\stackrel{0}{0} \\
\stackrel{+}{\#} \\
\stackrel{0}{0}\end{array}$ & $\begin{array}{l}\hat{N} \\
\dot{0} \\
\dot{H} \\
\dot{\sim} \\
\dot{v}\end{array}$ & $\begin{array}{l}\stackrel{0}{0} \\
\stackrel{+}{\pi} \\
\stackrel{0}{0}\end{array}$ & 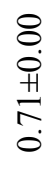 & $\begin{array}{l}\ddot{0} \\
0 \\
0 \\
\stackrel{+}{R} \\
0\end{array}$ & 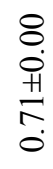 \\
\hline L. camara & 25 & 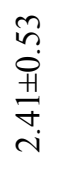 & $\begin{array}{l}n \\
0 \\
0 \\
+1 \\
\\
0\end{array}$ & 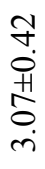 & $\begin{array}{l}n \\
0 \\
0 \\
\text { 10 } \\
0 \\
0\end{array}$ & 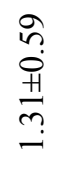 & 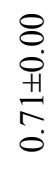 \\
\hline T. vogelii & 25 & 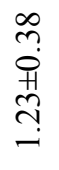 & $\begin{array}{l}8 \\
\ddot{0} \\
+ \\
\frac{1}{0} \\
\dot{0}\end{array}$ & 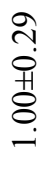 & $\begin{array}{l}\text { oे } \\
\dot{0} \\
+ \\
\infty \\
0 \\
0\end{array}$ & 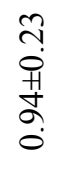 & $\begin{array}{l}n \\
0 \\
0 \\
+1 \\
0 \\
0\end{array}$ \\
\hline Deltamethrin & 0.04 & 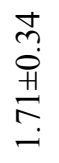 & $\begin{array}{l} \pm \\
\dot{0} \\
+ \\
⿱ 1 \\
0 \\
0\end{array}$ & 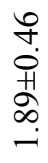 & $\begin{array}{l}8 \\
\dot{0} \\
\text { H } \\
\frac{1}{2} \\
\dot{0}\end{array}$ & 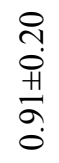 & $\begin{array}{l}\qquad \\
0 \\
0 \\
+1 \\
0 \\
\dot{0}\end{array}$ \\
\hline LSD (5\%) & & 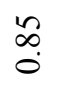 & $\begin{array}{l}\forall \\
\dot{0}\end{array}$ & $\stackrel{乛}{\circ}$ & $\frac{J}{0}$ & $\stackrel{\sim}{+}$ & $\frac{1}{0}$ \\
\hline
\end{tabular}

Values are mean $( \pm$ SEM $)(n=3)$. LSD values are applicable along the column. Delthamethrin $(0.81 /$ ha $)$, $\mathrm{DBML}=$ Diamondback moth larva, DMBA $=$ Diamondback-moth Adult, HAS $=$ Hours after spraying 
Table 3. Effect of botanicals on Diamondback moth (Plutella xylostella) population at $3^{\text {rd }}$ spraying.

\begin{tabular}{|c|c|c|c|c|c|c|c|}
\hline TREATMENTS & CONCENTRATIONS & & & & & & \\
\hline & $(\%)$ & 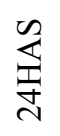 & $\begin{array}{l}\text { 岌 } \\
\text { 文 }\end{array}$ & 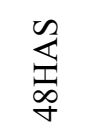 & $\begin{array}{l}\underset{4}{4} \\
\substack{\infty \\
+}\end{array}$ & $\underset{\mathbb{N}}{\stackrel{N}{\mathbb{N}}}$ & $\begin{array}{l}\underset{\mathbb{S}}{\mathbb{N}} \\
\underset{\mathrm{N}}{\mathrm{N}}\end{array}$ \\
\hline & & $\underset{\stackrel{m}{A}}{\vec{\rho}}$ & 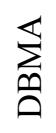 & 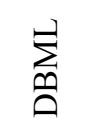 & 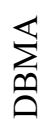 & $\sum_{\stackrel{D}{A}}^{B}$ & 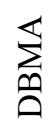 \\
\hline Control & Unsprayed & 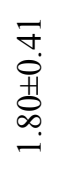 & $\begin{array}{l}8 \\
8 \\
0 \\
\stackrel{+}{*} \\
\stackrel{0}{0}\end{array}$ & 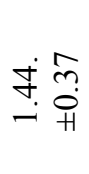 & 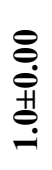 & $\begin{array}{l}\text { f } \\
\text { o } \\
\text { in } \\
\end{array}$ & 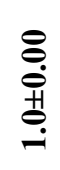 \\
\hline Z. officinales & 5 & $\begin{array}{l}\infty \\
\dot{0} \\
\dot{+} \\
\infty \\
i \\
i\end{array}$ & 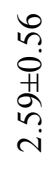 & $\begin{array}{l}\stackrel{a}{0} \\
\dot{0} \\
+1 \\
\infty \\
0 \\
0\end{array}$ & 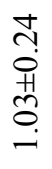 & 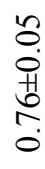 & $\begin{array}{l}n \\
0 \\
0 \\
0 \\
0 \\
0 \\
0\end{array}$ \\
\hline L. camara & 5 & $\begin{array}{l}\infty \\
\overrightarrow{0} \\
+ \\
0 \\
\\
i\end{array}$ & $\begin{array}{l}8 \\
8 \\
0 \\
\stackrel{+}{*} \\
\stackrel{0}{0}\end{array}$ & 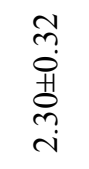 & $\begin{array}{l}0 \\
0 \\
0 \\
+1 \\
0 \\
0\end{array}$ & 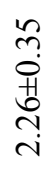 & $\begin{array}{l}\Xi \\
\pm \\
\dot{H} \\
\infty \\
0 \\
0\end{array}$ \\
\hline T. vogelii & 5 & $\begin{array}{l}\hat{\sigma} \\
\dot{0} \\
\ddot{H} \\
\sigma \\
-\end{array}$ & $\begin{array}{l}8 \\
\stackrel{8}{0} \\
+1 \\
\stackrel{+}{0} \\
0\end{array}$ & 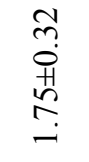 & $\begin{array}{l}n \\
0 \\
0 \\
+1 \\
0 \\
0\end{array}$ & $\begin{array}{l}\stackrel{m}{\stackrel{0}{\circ}} \\
\stackrel{+}{+} \\
\tilde{n}\end{array}$ & $\begin{array}{l}8 \\
\dot{0} \\
\stackrel{+}{\pi} \\
\stackrel{0}{0}\end{array}$ \\
\hline Z. officinales & 10 & 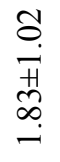 & 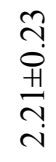 & $\begin{array}{l}8 \\
\dot{0} \\
+1 \\
\stackrel{+}{0} \\
0\end{array}$ & 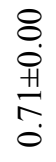 & 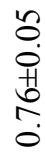 & $\begin{array}{l}8 \\
8 \\
0 \\
\stackrel{+}{7} \\
\ddot{0}\end{array}$ \\
\hline L. camara & 10 & $\begin{array}{l}\overrightarrow{0} \\
\dot{+} \\
\stackrel{+}{\circ} \\
\stackrel{-}{-}\end{array}$ & 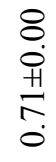 & $\begin{array}{l}\vec{\infty} \\
\dot{0} \\
\stackrel{+}{n} \\
\stackrel{n}{-}\end{array}$ & $\begin{array}{l}\infty \\
0 \\
0 \\
0 \\
0 \\
\infty \\
0 \\
0\end{array}$ & 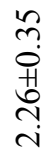 & $\begin{array}{l}n \\
0 \\
0 \\
0 \\
0 \\
0 \\
0 \\
0\end{array}$ \\
\hline T. vogelii & 10 & $\begin{array}{l}\hat{n} \\
0 \\
+ \\
\hat{+} \\
\text { a }\end{array}$ & $\begin{array}{l}8 \\
\dot{0} \\
\stackrel{+}{+} \\
+ \\
0 \\
0\end{array}$ & 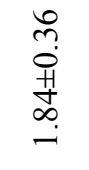 & $\begin{array}{l}8 \\
\dot{0} \\
\stackrel{+}{7} \\
\stackrel{0}{0}\end{array}$ & 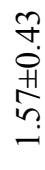 & $\begin{array}{l}8 \\
\stackrel{0}{0} \\
\stackrel{+1}{r} \\
\stackrel{0}{0}\end{array}$ \\
\hline Z.. officinales & 25 & $\begin{array}{l}\stackrel{+}{n} \\
\text { H. } \\
\infty \\
\infty \\
\dot{n}\end{array}$ & $\begin{array}{l}\circ \\
\infty \\
0 \\
0 \\
\infty \\
\infty \\
\dot{n}\end{array}$ & 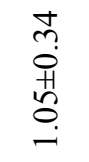 & 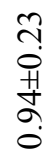 & $\begin{array}{l}8 \\
0 \\
\text { +1 } \\
\text { i. } \\
0\end{array}$ & 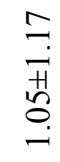 \\
\hline
\end{tabular}




\begin{tabular}{|c|c|c|c|c|c|c|c|}
\hline L. camara & 25 & 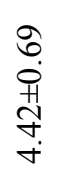 & $\begin{array}{l}\qquad \\
0 \\
0 \\
+1 \\
\stackrel{0}{0} \\
0\end{array}$ & $\begin{array}{l}\hat{n} \\
\dot{0} \\
\text { +1 } \\
\stackrel{n}{n} \\
\dot{m}\end{array}$ & $\begin{array}{l}\infty \\
0 \\
0 \\
0 \\
0 \\
0 \\
0 \\
0\end{array}$ & $\begin{array}{l}\stackrel{0}{0} \\
\text { in } \\
\text { ले } \\
\text { ले }\end{array}$ & $\begin{array}{l}\overrightarrow{+} \\
0 \\
+1 \\
0 \\
ت\end{array}$ \\
\hline T. vogelii & 25 & $\begin{array}{l}\infty \\
\infty \\
0 \\
\dot{1} \\
\infty \\
\infty \\
-\end{array}$ & $\begin{array}{l}n \\
0 \\
0 \\
+1 \\
0 \\
0 \\
0\end{array}$ & 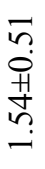 & 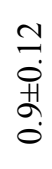 & $\begin{array}{l}? \\
\stackrel{0}{0} \\
\text { 웅 } \\
\stackrel{5}{-}\end{array}$ & 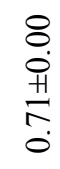 \\
\hline Deltamethrin & 0.04 & $\begin{array}{l}\stackrel{+}{0} \\
\dot{0} \\
\stackrel{+}{m} \\
\dot{m}\end{array}$ & 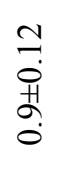 & $\begin{array}{l}\vec{\circ} \\
\stackrel{\circ}{+} \\
\stackrel{H}{n} \\
\vec{i}\end{array}$ & $\begin{array}{l}\text { I } \\
\stackrel{0}{0} \\
\dddot{1} \\
\vdots\end{array}$ & 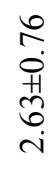 & \begin{tabular}{l}
8 \\
0 \\
0 \\
\multirow{1}{*}{} \\
0
\end{tabular} \\
\hline LSD (5\%) & & $\stackrel{?}{n}$ & $\frac{1}{0}$ & $\stackrel{n}{\rightleftarrows}$ & $\hat{0}$ & $\stackrel{m}{=}$ & $\stackrel{ \pm}{\sim}$ \\
\hline
\end{tabular}

Values are mean $( \pm \operatorname{SEM})(\mathrm{n}=3)$. LSD values are applicable along the column. Delthamethrin $(0.8 \mathrm{l} / \mathrm{ha})$, $\mathrm{DBML}=$ Diamondback moth larva, DMBA $=$ Diamondback-moth Adult, HAS $=$ Hours after spraying

Table 4. Effect of botanicals on Diamondback moth (Plutella xylostella) population at $4^{\text {th }}$ spraying.

\begin{tabular}{|c|c|c|c|c|c|c|c|}
\hline TREATMENTS & CONCENTRATIONS & & & & & & \\
\hline & $(\%)$ & 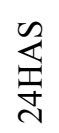 & 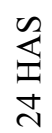 & $\underset{\substack{\infty \\
+}}{\substack{++}}$ & 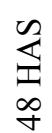 & $\underset{\mathbb{N}}{\stackrel{\mathbb{N}}{\mathbb{N}}}$ & $\begin{array}{l}\mathbb{Z}_{\mathbf{S}} \\
\text { I } \\
\mathbb{N}\end{array}$ \\
\hline & & $\underset{⿱ 亠 䒑}{\stackrel{m}{D}}$ & 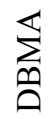 & 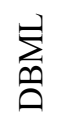 & 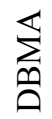 & 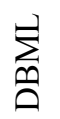 & 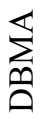 \\
\hline Control & Unsprayed & 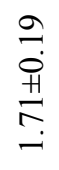 & \begin{tabular}{l}
8 \\
$\dot{0}$ \\
0 \\
\multirow{0}{*}{} \\
0
\end{tabular} & $\begin{array}{l}\vec{N} \\
0 \\
0 \\
0 \\
0 \\
-\end{array}$ & $\begin{array}{l}8 \\
\dot{0} \\
\stackrel{+}{7} \\
\stackrel{2}{0}\end{array}$ & $\begin{array}{l}\infty \\
\text { ñ } \\
\stackrel{+}{+} \\
\hat{n} \\
\stackrel{n}{n}\end{array}$ & $\begin{array}{l}8 \\
\dot{0} \\
\stackrel{+}{+} \\
\frac{1}{0}\end{array}$ \\
\hline Z. officinales & 5 & 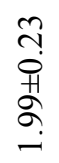 & 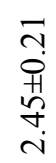 & $\begin{array}{l}0 \\
0 \\
0 \\
+1 \\
0 \\
0\end{array}$ & 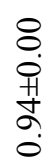 & $\begin{array}{l}8 \\
\stackrel{8}{0} \\
\frac{\#}{0} \\
0\end{array}$ & $\begin{array}{l}8 \\
\stackrel{8}{0} \\
\frac{1}{2} \\
\stackrel{0}{0}\end{array}$ \\
\hline L. camara & 5 & $\begin{array}{l}\bar{n} \\
0 \\
0 \\
8 \\
i \\
i\end{array}$ & 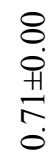 & $\begin{array}{l}\bar{n} \\
0 \\
\text { ஸे } \\
0 \\
i\end{array}$ & 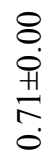 & $\begin{array}{l}\hat{n} \\
0 \\
+1 \\
\hat{n} \\
\stackrel{1}{0}\end{array}$ & $\begin{array}{l}\stackrel{8}{0} \\
\stackrel{0}{+} \\
\stackrel{1}{*} \\
0\end{array}$ \\
\hline
\end{tabular}




\begin{tabular}{|c|c|c|c|c|c|c|c|}
\hline T. vogelii & 5 & $\begin{array}{l}0 \\
\stackrel{n}{0} \\
\stackrel{+}{H} \\
\stackrel{1}{\infty} \\
\infty\end{array}$ & $\begin{array}{l}8 \\
8 \\
\stackrel{+}{1} \\
\stackrel{1}{*} \\
0\end{array}$ & 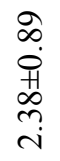 & $\begin{array}{l}8 \\
\stackrel{8}{0} \\
\stackrel{+}{\#} \\
\dot{0}\end{array}$ & 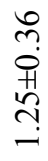 & 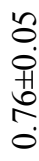 \\
\hline Z. officinales & 10 & 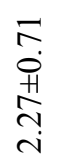 & $\begin{array}{l}0 \\
0 \\
0 \\
0 \\
0 \\
\end{array}$ & $\begin{array}{l}n \\
0 \\
0 \\
+1 \\
0 \\
0\end{array}$ & $\begin{array}{l}8 \\
\stackrel{0}{0} \\
\stackrel{+}{\#} \\
\stackrel{0}{0}\end{array}$ & $\begin{array}{l}8 \\
\stackrel{8}{0} \\
\stackrel{+}{*} \\
\stackrel{1}{0}\end{array}$ & 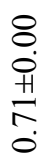 \\
\hline L. camara & 10 & $\begin{array}{l}\stackrel{0}{a} \\
\dot{H} \\
\stackrel{H}{n} \\
\stackrel{i}{n}\end{array}$ & $\begin{array}{l}8 \\
\stackrel{0}{0} \\
\stackrel{+}{\#} \\
\stackrel{0}{0}\end{array}$ & $\begin{array}{l}\bar{m} \\
0 \\
+1 \\
0 \\
0 \\
\dot{i}\end{array}$ & $\begin{array}{l}8 \\
0 \\
\stackrel{+}{1} \\
\stackrel{1}{*} \\
\dot{0}\end{array}$ & 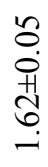 & $\begin{array}{l}8 \\
\stackrel{0}{0} \\
+1 \\
\frac{1}{0}\end{array}$ \\
\hline T. vogelii & 10 & 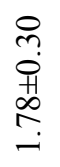 & $\begin{array}{l}8 \\
0 \\
0 \\
\stackrel{+}{1} \\
0 \\
0\end{array}$ & 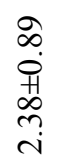 & 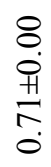 & 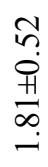 & 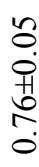 \\
\hline Z. officinales & 25 & $\begin{array}{l}0 \\
\vdots \\
0 \\
+1 \\
\stackrel{1}{*} \\
-\end{array}$ & $\begin{array}{l}\infty \\
\stackrel{H}{H} \\
\stackrel{H}{\sim}\end{array}$ & $\begin{array}{l}8 \\
\dot{0} \\
+1 \\
i \\
\dot{0}\end{array}$ & 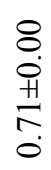 & 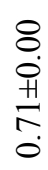 & $\begin{array}{l}m \\
\stackrel{m}{0} \\
+1 \\
+ \\
0\end{array}$ \\
\hline L. camara & 25 & $\begin{array}{l}\tilde{\sigma} \\
\stackrel{0}{0} \\
\stackrel{+}{1} \\
\tilde{O} \\
\dot{+}\end{array}$ & $\begin{array}{l}8 \\
\dot{0} \\
\stackrel{+}{*} \\
\dot{0}\end{array}$ & $\begin{array}{l}\nexists \dot{J} \\
\stackrel{+}{+} \\
\stackrel{⿱}{r} \\
\dot{r}\end{array}$ & $\begin{array}{l}8 \\
\dot{0} \\
\stackrel{+}{1} \\
\stackrel{1}{\circ} \\
\dot{0}\end{array}$ & $\begin{array}{l}0 \\
\text { ?’ } \\
\stackrel{+}{+} \\
\infty \\
\dot{m}\end{array}$ & $\begin{array}{l}n \\
0 \\
0 \\
0 \\
0 \\
0 \\
0\end{array}$ \\
\hline T. vogelii & 25 & 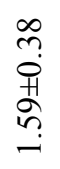 & $\begin{array}{l}8 \\
0 \\
\dot{H} \\
\frac{H}{0} \\
\ddot{0}\end{array}$ & $\begin{array}{l}\bar{n} \\
0 \\
\text { Ḣ } \\
0 \\
\dot{i}\end{array}$ & $\begin{array}{l}8 \\
0 \\
0 \\
+1 \\
0 \\
0\end{array}$ & 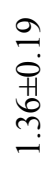 & $\begin{array}{l}0 \\
0 \\
0 \\
0 \\
0 \\
0\end{array}$ \\
\hline Deltamethrin & 0.04 & $\begin{array}{l}\hat{n} \\
0 \\
+1 \\
0 \\
\dot{0} \\
\dot{n}\end{array}$ & 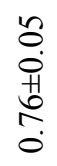 & $\begin{array}{l}\hat{n} \\
0 \\
+1 \\
\infty \\
n \\
n \\
n\end{array}$ & $\begin{array}{l}8 \\
\dot{0} \\
\dot{+} \\
\stackrel{1}{*} \\
\dot{0}\end{array}$ & $\begin{array}{l}\stackrel{+}{+} \\
\stackrel{+}{+} \\
\frac{1}{n} \\
\text { r }\end{array}$ & $\begin{array}{l}0 \\
0 \\
0 \\
0 \\
0 \\
0\end{array}$ \\
\hline LSD $(5 \%)$ & & $\stackrel{8}{\circ}$ & $\begin{array}{l}n \\
\infty \\
0\end{array}$ & $\frac{R}{0}$ & $\frac{m}{0}$ & $\begin{array}{l}+ \\
0 \\
0\end{array}$ & $\stackrel{0}{\circ}$ \\
\hline
\end{tabular}

Values are mean $( \pm$ SEM) $(n=3)$. LSD values are applicable along the column. Delthamethrin $(0.81 /$ ha $)$, $\mathrm{DBML}=$ Diamondback moth larva, DMBA $=$ Diamondback-moth Adult, HAS $=$ Hours after spraying.

Table 5. Effect of botanicals on plant height of cabbage after spraying.

\begin{tabular}{|c|c|c|c|c|c|}
\hline \multirow[t]{2}{*}{ TREATMENTS } & CONCENTRATIONS & \multicolumn{4}{|c|}{ WEEKS AFTETR SPRAYING } \\
\hline & $\%$ & $\begin{array}{l}\vec{y} \\
\frac{1}{1} \\
\frac{1}{3}\end{array}$ & $\frac{N}{\frac{N}{y}}$ & $\begin{array}{l}m \\
\frac{m}{y} \\
3\end{array}$ & 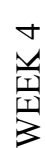 \\
\hline
\end{tabular}




\begin{tabular}{|c|c|c|c|c|c|}
\hline Control & Unsprayed & $\begin{array}{l}\bar{i} \\
\stackrel{+}{+} \\
\stackrel{\tilde{O}}{\Xi} \\
=\end{array}$ & \begin{tabular}{l}
8 \\
i \\
H \\
\multirow{n}{*}{} \\
$\stackrel{n}{=}$
\end{tabular} & 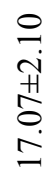 & $\begin{array}{l}\overrightarrow{7} \\
\dot{0} \\
+1 \\
\tilde{n} \\
\stackrel{1}{0}\end{array}$ \\
\hline Z. officinales & 5 & $\begin{array}{l}m \\
\stackrel{+}{N} \\
+1 \\
+ \\
\dot{0}\end{array}$ & $\begin{array}{l}\stackrel{8}{i} \\
\text { 茂 } \\
\stackrel{i}{i}\end{array}$ & 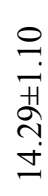 & $\begin{array}{l}\infty \\
\infty \\
0 \\
0 \\
+1 \\
n \\
n\end{array}$ \\
\hline L. camara & 5 & $\begin{array}{l}\stackrel{?}{\cdot} \\
\stackrel{H}{+} \\
\stackrel{+}{0} \\
\stackrel{0}{0}\end{array}$ & 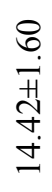 & 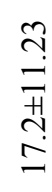 & $\begin{array}{l}\stackrel{+}{+} \\
\dot{H} \\
\dot{H} \\
\infty \\
\infty\end{array}$ \\
\hline T. vogelii & 5 & 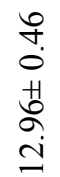 & 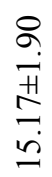 & 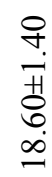 & $\begin{array}{l}\text { ㅇ. } \\
\text { H. } \\
\dot{\infty} \\
\infty\end{array}$ \\
\hline Z. officinales & 10 & 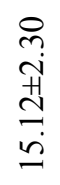 & 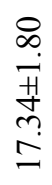 & 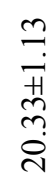 & 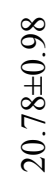 \\
\hline L. camara & 10 & 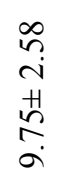 & 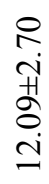 & $\begin{array}{l}\stackrel{0}{-} \\
\stackrel{H}{1} \\
\infty \\
0 \\
0\end{array}$ & 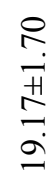 \\
\hline T. vogelii & 10 & $\begin{array}{l}\infty \\
\stackrel{\infty}{+} \\
\stackrel{+}{a} \\
\stackrel{ \pm}{ \pm}\end{array}$ & 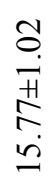 & 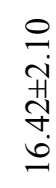 & 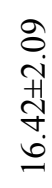 \\
\hline Z. officinales & 25 & 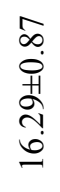 & $\begin{array}{l}0 \\
\stackrel{0}{0} \\
0 \\
0 \\
0 \\
\infty\end{array}$ & 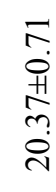 & 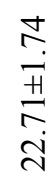 \\
\hline L. camara & 25 & $\begin{array}{l}+ \\
\infty \\
0 \\
⿱ 艹 \\
n \\
n \\
=\end{array}$ & 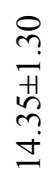 & 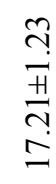 & $\begin{array}{l}\mathbb{8} \\
\stackrel{i}{0} \\
\stackrel{H}{\infty} \\
\dot{\pi}\end{array}$ \\
\hline
\end{tabular}




\begin{tabular}{|c|c|c|c|c|c|}
\hline T. vogelii & 25 & 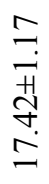 & $\begin{array}{l}\dot{+} \\
+ \\
+ \\
\sigma \\
\sigma \\
\infty \\
-\end{array}$ & 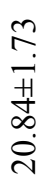 & 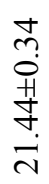 \\
\hline Delthamethrin & 0.04 & $\begin{array}{l}0 \\
a \\
i \\
+1 \\
i n \\
\end{array}$ & 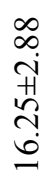 & 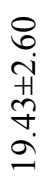 & 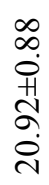 \\
\hline L.S.D & & $\frac{m}{6}$ & ڤn & $\begin{array}{l}\text { ஜீ } \\
\dot{n}\end{array}$ & $\begin{array}{l}8 \\
\stackrel{0}{0}\end{array}$ \\
\hline
\end{tabular}

Values are mean $( \pm \mathrm{SEM})(\mathrm{n}=3)$. LSD values are applicable along the column. Delthamethrin $(0.81 / \mathrm{ha})$.

Table 6. Effect of botanicals on leaf damage of cabbage after spraying.

\begin{tabular}{|c|c|c|c|c|c|}
\hline \multirow[t]{2}{*}{ TREATMENTS } & \multirow{2}{*}{$\begin{array}{c}\text { CONCENTRATIONS } \\
\qquad \begin{array}{c}\% \\
\%\end{array}\end{array}$} & \multicolumn{4}{|c|}{ WEEKS AFTETR SPRAYING } \\
\hline & & $\frac{\vec{y}}{\frac{1}{1}}$ & $\frac{\sqrt{u}}{\frac{1}{N}}$ & $\frac{m}{\frac{n}{y}}$ & $\begin{array}{l}\dot{\forall} \\
\frac{\Delta}{\mid} \\
\frac{1}{3}\end{array}$ \\
\hline Control & Unsprayed & $\begin{array}{l}\infty \\
\stackrel{\infty}{I} \\
\stackrel{+1}{2} \\
\infty \\
\dot{0} \\
i\end{array}$ & 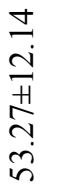 & 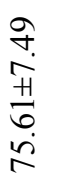 & 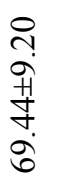 \\
\hline Z. officinales & 5 & 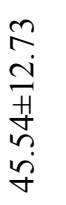 & 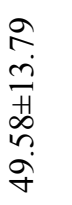 & $\begin{array}{l}0 \\
n \\
i n \\
\text { in } \\
i \\
\infty \\
n\end{array}$ & 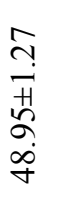 \\
\hline L. camara & 5 & 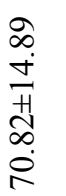 & 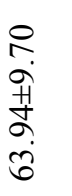 & $\begin{array}{l}\stackrel{8}{0} \\
\stackrel{1}{1} \\
\infty \\
i \\
i \\
i\end{array}$ & 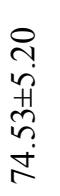 \\
\hline T. vogelii & 5 & $\begin{array}{l}\stackrel{+}{2} \\
\infty \\
+ \\
\infty \\
\infty \\
\infty\end{array}$ & 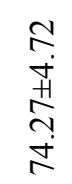 & 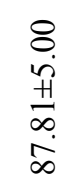 & $\begin{array}{l}n \\
\stackrel{n}{\oplus} \\
\ddot{H} \\
\infty \\
\infty\end{array}$ \\
\hline
\end{tabular}




\begin{tabular}{|c|c|c|c|c|c|}
\hline Z.officinales & 10 & 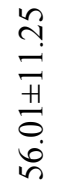 & $\begin{array}{l}\stackrel{0}{0} \\
\text { iे } \\
\text { ले } \\
\text { ம் }\end{array}$ & $\begin{array}{l}\tilde{n} \\
\text { H. } \\
\text { o. } \\
\dot{8}\end{array}$ & 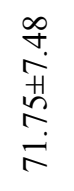 \\
\hline L. camara & 10 & 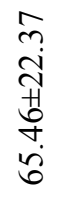 & 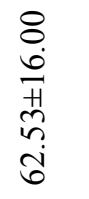 & 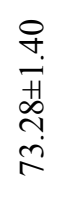 & $\begin{array}{l}\stackrel{R}{0} \\
\dot{0} \\
+ \\
\stackrel{+}{n} \\
\stackrel{1}{*}\end{array}$ \\
\hline T.vogelii & 10 & 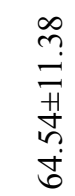 & 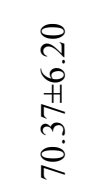 & 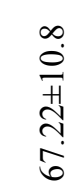 & $\begin{array}{l}8 \\
\stackrel{9}{+} \\
\stackrel{+}{0} \\
\text { in }\end{array}$ \\
\hline Z. officinales & 25 & $\begin{array}{l}\infty \\
0 \\
0 \\
0 \\
+ \\
0 \\
0 \\
0 \\
0\end{array}$ & $\begin{array}{l}\stackrel{8}{\circ} \\
\infty \\
+1 \\
\infty \\
\infty \\
\infty \\
\infty \\
\infty\end{array}$ & $\begin{array}{l}\stackrel{n}{n} \\
i+n \\
\hat{n} \\
i n \\
r\end{array}$ & 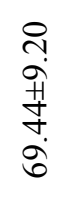 \\
\hline L. camara & 25 & 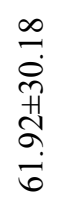 & 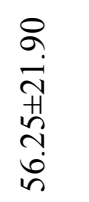 & $\begin{array}{l}8 \\
0 \\
\infty \\
0 \\
0 \\
\tilde{n} \\
\tilde{n} \\
\tilde{n}\end{array}$ & $\begin{array}{l}\text { ले } \\
\text { के } \\
\text { ले } \\
\ddot{0}\end{array}$ \\
\hline T. vogelii & 25 & 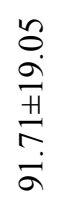 & 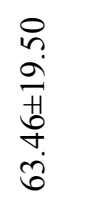 & 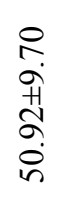 & 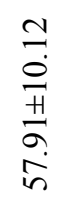 \\
\hline Delthamethrin & 0.04 & 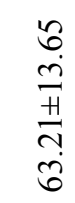 & 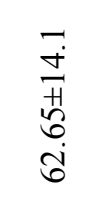 & $\begin{array}{l}8 \\
\dot{8} \\
i \\
\forall \\
+ \\
\dot{H} \\
\dot{n}\end{array}$ & 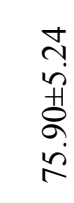 \\
\hline L.S.D & & 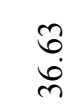 & $\begin{array}{l}\text { ले } \\
\ddot{d}\end{array}$ & 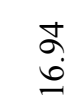 & ڤ્nે \\
\hline
\end{tabular}

Values are mean $( \pm$ SEM) $(n=3)$. LSD values are applicable along the column. Delthamethrin $(0.81 /$ ha $)$. 
Table 7. Effect of botanicals on yield and head damage of cabbage.

\begin{tabular}{|c|c|c|c|}
\hline TREATMENTS & CONCENTRATIONS \% & YIELD Kg/ha & HEAD DAMAGE \% \\
\hline Control & Unsprayed & $0.28 \pm 0.02$ & $1.41 \pm 0.22$ \\
\hline Z. officinales & 5 & $0.19 \pm 0.06$ & $1.66 \pm 0.30$ \\
\hline L. camara & 5 & $0.21 \pm 0.12$ & $2.00 \pm 0.28$ \\
\hline T.vogelii & 5 & $0.24 \pm 0.07$ & $1.50 \pm 0.14$ \\
\hline Z. officinales & 10 & $0.31 \pm 0.06$ & $1.66 \pm 0.08$ \\
\hline L. camara & 10 & $0.38 \pm 0.22$ & $1.41 \pm 0.41$ \\
\hline T. vogelii & 10 & $0.24 \pm 0.08$ & $1.83 \pm 0.14$ \\
\hline Z. officinales & 25 & $0.50 \pm 0.13$ & $1.50 \pm 0.25$ \\
\hline L. camara & 25 & $0.29 \pm 0.08$ & $1.25 \pm 0.14$ \\
\hline T. vogelii & 25 & $0.23 \pm 0.39$ & $1.33 \pm 0.44$ \\
\hline Delthamethrin & 0.04 & $0.28 \pm 0.08$ & $1.83 \pm 0.36$ \\
\hline L S D (5\%) & & 0.14 & 0.25 \\
\hline
\end{tabular}

Values are mean $( \pm \mathrm{SEM})(\mathrm{n}=3)$. LSD values are applicable along the column. Delthamethrin $(0.8 \mathrm{l} / \mathrm{ha})$.

\section{DISCUSSION AND CONCLUSIONS}

Among the plant extracts, $Z$. officinales extract proved effective in controlling $P$. xylostella larva at different concentration but gave the best at $25 \%$ concentrations. Insect population decreased as exposure hours increased and when compared with synthetic insecticides, it performed better. This agrees with the work of Dadang et al. (2009) who reported that Piper retrofractum and Annona squamosa extracts were more effective than synthetic insecticides (Deltamethrine) in controlling Crocidolomia pavonana (F.) and $P$. xylostella (L.) which are insect pests of cabbage. Prakash Rao (1997) and Sridhar et al (2002) reported that the active ingredients in $Z$. officinale which are pungent makes it a very good insecticides with repellent mode of action against insect pests such as aphids, leaf miner, bollworm and thrips. Gingerols and shogaols are the active insecticidal materials in $Z$. officinale rhizome (Goto et al., 1990).

Conversely, it was observed from this study that $Z$. officnales was ineffective against $P$. xylostella adult and as its concentrations increased the population of $P$. xylostella adult increased. This shows that it does not have effect in controlling adult insect population of cabbage. This corroborates with the work of Hala et al. (2010) who reported that plant extracts of $Z$. officinale showed good level of efficiency against nymphs of Bemisia tabaci and Aphis craccivora. Dadang et al. (2007) reported that extract of Aglaia odorata was effective in controlling several agricultural insect pests including $P$. xylostella and Crocidolomia pavonana larvae. 
Deltamethrin and L. camara - treated plots had higher population of $P$. xylostella larva compared with the other insect at different concentrations and HAS. This showed that $L$. camara was ineffective as Deltamethrin in controlling the P. xylostella larva. Report shows that synthetic insecticides do not have much effect in reducing insect population but rather increased. Ninsin (1997) observed that P. xylostella was resistant to many conventional insecticides and so spraying DBM infested cabbage plot often has little effect on the pest. Youdeowei (1988) and Shelton (1993) reported that DBM can cause serious damage even with application of several different insecticides because of its ability to develop resistance to almost all insecticides.

Prevalence of leave damage pointed out that as the concentrations increases in $T$. vogelii, L. camara leaves damage reduced. This corroborates the work of Isirima (2010) that leaf damage decreased with increasing application of the plant extracts (garlic and ginger). Throughout the period of this study the effectiveness of the extracts of $Z$. officinales kept on decreasing with increase in the time after application. This may be due to photo - degradation of its bioactive composition. It is recommended that extract of $Z$. officinales at $25 \%$ concentration should be evaluated for its bioactive against other field insect pests of cabbage in order to establish its spectrum of activity.

\section{Acknowledgements}

The authors wish to acknowledge Dr. S.A Babarinde, Department of Crop and Environmental Protection Ladoke Akintola University of Technology Ogbomoso, Nigeria.

\section{References}

[1] Adebayo T. A. (2003). Efficacy of mixture formulations of synthesis and botanical insecticides in the control of insect pests of okra and cowpea. Ph.D. Thesis submitted to Ladoke Akintola University of Technology, Ogbomoso, Nigeria.

[2] Dadang D. P., Soekarno B. P. W., Winasa I. W., Hindayana D., Munif A. (2003a). Indoxy farmer survey in Brebes and Tegal, Central Java. Report. Bogor: Department of Plant Pests and Diseases, IPB.

[3] Dadang Fitriasari Eva Dwi, Djoko P., ISSAAS 15(1) (2009) 42-51.

[4] Devlin J. F., Zettel T. (1999). Ecoagriculturee : initiatives in eastern and southern Africa. Weaver Press.

[5] Duke S. O. (1990). Natural pesticides from plants. In Janick J and simon J.E Eds. Advances in new crops. Timber Press, Portland, PP 511-517.

[6] Goto C., Kasuya S., Koga K., Ohtomo H., Kagei N., Parasitology Reseash 76 (1990) 653-656.

[7] Hala M., Abou Y., Sayedaf F., Howaida M. T., World Journal of Agricultural Science 6(4) (2010) 434-439.

[8] Jagerstad M., Jastrebova J., Svensson, Wiss. u.-Technol. 37 (2002) 603-611.

[9] Kyung Y. Y., Woodams E., Hang Y. D., Bioresource Technology 97 (2006) 1427-1430. 
[10] Meadows A. B. (1988). Ginger processing for food and for industry proceedings of the first National Ginger Workshop, Umudike, p. 34-42.

[11] Ninsin D. K. (1997). Insecticides use pattern and residue levels in cabbage cultivated within the Acera-Tem metropolitan of Ghana unpublished master of Philosophy thesis. Insect Science Programme. University of Ghana.

[12] Oparake A. M., Journal of Plant Protection Research: 45 (2005) 1-6.

[13] Owolade O. F., Alabi B. S., Osikanlu Y. O. K., Odeyemi O. O., Food and Agricultural Environment 2 (2005) 237-240.

[14] Prakash A., Rao J., Nandagopal V., Journal of Biopesticides 1(2) (2008) 154-169.

[15] Rodriguez R., Jimenez A., Fernandez J., Guillen R., Heredia A., Trends in Food Science \& Technology 17 (2006) 3-15.

[16] Saharaya and Mungesan (1998). An Introduction to Insect Pest and their control. Macmillan Publishers Ltd. New York pp 97.

[17] Shelton A. M., Wyman J. A., Cushing N. L., Apfelbeck K., Dennehy T. J., Mah S. E. R., Eigenbrode S. D., Journal Economic Entomology 86 (1993) 11-19.

[18] Sridhar S., Arumugasamy S., Sarawathy H., Vijayadakshni K. (2002). Organic Vegetable Gardening Centre for India Knowledge System Chennai. India.

[19] Tabashmik B. E., Cushing N. L., Johnson M. W., Journal of Economic Entomology 80 (1987) 11091-1099.

[20] Taleker N. S. (1992). Management of diamondback moth and other crucifer pest in Proceedings of International Workshop. AVRDC, Shanhua, Taiwan.

[21] Talekar N. S., Shelton A. M., Annual Review of Entomology 38 (1993) 275-301.

[22] Talaker W. S. (2000). Chinese cabbage Proceedings of the first international symposium on Chinese Cabbge AVRDC Shanhua, Taiwan pp. 67-69.

[23] USDA (2009). National Nutrient Data base for standard Reference.

[24] Youdewei A. (1988). Aspects of the economic impact of insect pests and Pest management on crops production in Nigeria. Paper delivered to the agro chemicals Group Manufacturers Association of Nigeria, Sheraton Hotel, Lagos. 